\title{
Cerebellar Development in the Preterm Neonate: Effect of Supratentorial Brain Injury
}

\author{
EMILY W. Y. TAM, DONNA M. FERRIERO, DUAN XU, JEFFREY I. BERMAN, DANIEL B. VIGNERON, \\ A. JAMES BARKOVICH, AND STEVEN P. MILLER
}

\begin{abstract}
Departments of Neurology and Pediatrics [E.W.Y.T., D.M.F., S.P.M.], and Radiology and Biomedical Imaging [D.X., J.I.B., D.B.V., A.J.B.], University of California, San Francisco, San Francisco, California 94143; Department of Pediatrics [S.P.M.], University of British Columbia, Vancouver, Canada V6H 3 V4
\end{abstract}

\begin{abstract}
Cerebellar injury has been increasingly recognized as a complication of preterm birth, with decreased cerebellar volumes seen on follow-up neuroimaging. A cohort of 38 preterm newborns, including 14 with two scans, was studied with MRI, including single-shot fast spin-echo diffusion tensor imaging (DTI) sequence specifically to assess the posterior fossa. Early changes in the cerebellum [apparent diffusion coefficient (ADC) and fractional anisotropy (FA)] were assessed and correlated with supratentorial manifestations of injury [intraventricular hemorrhage (IVH) or white matter injury (WMI)]. ADC decreased and FA increased with increasing gestational age in both cerebellar gray and white matter. Severe IVH was associated with increased ADC in the middle cerebellar peduncles and hila of the cerebellar nuclei, decreased ADC in the cerebellar cortex, and decreased FA in all three regions. Changes with WMI were not consistent. Significant developmental changes in water diffusion were seen in cerebellar gray and white matter that were altered in patients with supratentorial IVH. DTI studies may provide an early indicator for cerebellar injury and abnormal cerebellar development in preterm neonates. (Pediatr Res 66: 102-106, 2009)
\end{abstract}

$\mathrm{P}$ remature birth is a significant risk factor for adverse motor, coordination, cognitive, and behavioral outcomes in survivors. The cerebellum has traditionally been associated with motor tone and coordination and recently has been implicated in cognitive function (1-4). Decreased cerebellar volumes have been described in follow-up MRI studies of individuals born preterm $(5,6)$. A number of recent studies have performed volumetric analyses of structural neuroimaging studies, correlating decreased cerebellar volume with supratentorial injuryincluding white matter injury (WMI), intraventricular hemorrhage (IVH) with ventricular dilatation, and hemorrhagic parenchymal infarction - and neurodevelopmental outcome (7-9).

Although volumetric analyses in neonates born preterm have proven useful in analyzing conventional neuroimaging, diffusion tensor imaging (DTI) is a different approach to assessing the neonatal brain, focused on microstructural changes, often related

Received September 29, 2008; accepted February 2, 2009

Correspondence: Emily W. Y. Tam, MDCM, FRCPC, Department of Neurology, University of California, San Francisco, 521 Parnassus Ave., Room C-215, San Francisco, CA 94143-0663; e-mail: emily.tam@ucsf.edu

Supported by grants from the National Institutes of Health (R01 NS40117 and R01 NS046432), in part through the Pediatric Clinical Research Center, University of California, San Francisco, with funds provided by the National Center for Research Resources, 5 M01 RR-01271, U.S. Public Health Service, the Canadian Institute for Health Research, and the Michael Smith Foundation for Health Research. to injury, that can be detected early in the neonatal course. This ability to detect early changes may afford DTI the potential to aid in early prognosis of neurodevelopmental outcome. DTI studies have been used to identify maturation trends in cerebral (supratentorial) white matter even before myelination is evident $(10,11)$. Serial studies in preterm neonates show changes in diffusion anisotropy of supratentorial structures with age (12). However, magnetic susceptibility effects can cause significant distortions in the imaging of the posterior fossa using the echo planar (EPI) technique, limiting the ability to accurately characterize the cerebellum if parallel imaging is not available. Distortions in the imaging of cerebellar structures can be overcome using a novel single-shot fast spin-echo (SSFSE) DTI technique (13). We hypothesized that, using SSFSE-DTI, changes in diffusion parameters with age could be detected in the cerebellum and that these changes would be affected by supratentorial IVH and WMI.

\section{METHODS}

A cohort of preterm neonates born in 2002-2004 and admitted to our institution was studied with MRI, including SSFSE-DTI sequences. These patients were recruited as a part of an ongoing research protocol $(12,13)$. Prematurely born neonates $(<34$-wk gestational age) born in or transferred to our institution's NICU were approached for enrollment in a prospective cohort study evaluating the detection of brain injury by MRI. Gestational age was calculated based on the last menstrual period or early ultrasound ( $<24 \mathrm{wk})$. If the difference between the two methods were $>7 \mathrm{~d}$, the ultrasound date was used. Exclusion criteria for study enrollment were as follows: 1) clinical evidence of a congenital malformation or syndrome, 2) congenital TORCH infection, and 3) newborns too clinically unstable for transport to the MRI scanner. All eligible infants were considered for study, and parental consent was obtained for all cases according to a protocol approved by the UCSF Committee on Human Research. Clinical history, including the number of days intubated, presence of patent ductus arteriosus, documentation of infection, and clinical seizures, was recorded. A customized MR-compatible incubator with a specialized neonatal head coil was used to provide a quiet, temperature-controlled, well-monitored environment for the neonate, minimizing patient movement and improving the signal-to-noise ratio (14). SSFSE-DTI was acquired using repetition time (TR)/echo time (TE) $=$ $11 \mathrm{~s} / 92 \mathrm{~ms}$, six gradient directions, four averages, $b=600 \mathrm{~s} / \mathrm{mm}^{2}$, and a voxel size of $0.7 \times 0.7 \times 3 \mathrm{~mm}^{3}$ (13). In addition to SSFSE DTI, MR imaging of the brain included the following: 1) T1 weighted sagittal spin echo images (4-mm thickness) using repetition time $(\mathrm{TR})=500 \mathrm{~ms}$, echo time $(\mathrm{TE})=11 \mathrm{~ms}, 1$ excitation, and $192 \times 256$ acquisition matrix; 2) T2 weighted dual spin echo (4-mm thickness) with $\mathrm{TR}=3000 \mathrm{~ms}, \mathrm{TE}=60$ and $120 \mathrm{~ms}$, and $192 \times 256$ acquisition matrix; and 3) coronal 3D SPGR T1 weighted images (partition

\footnotetext{
Abbreviations: ADC, apparent diffusion coefficient; DTI, diffusion tensor imaging; FA, fractional anisotropy; IVH, intraventricular hemorrhage; WMI, white matter injury
} 


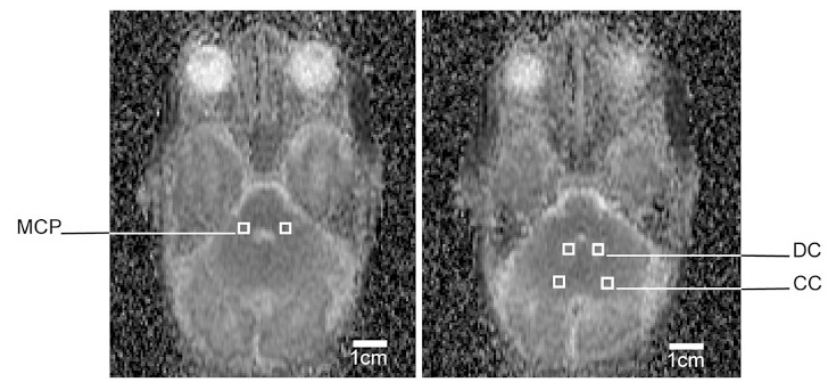

Figure 1. A representative SSFSE-DTI ADC map of the premature neonatal posterior fossa, showing the regions of interest in the middle cerebellar peduncles (MCP), deep cerebellar nuclei (DC), and cerebellar cortex (CC).

size $=1.5 \mathrm{~mm}$ ) with $\mathrm{TR}=36 \mathrm{~ms}, \mathrm{TE}=\min$, Flip angle $=35^{\circ}, \mathrm{NEX}=2$, $\mathrm{FOV}=18 \mathrm{~cm}$, as described previously (15).

Using this SSFSE-DTI sequence, spatially averaged apparent diffusion coefficient (ADC or mean diffusivity) and fractional anisotropy (FA) of the cerebellum were measured from $0.027 \mathrm{~cm}^{3}$ bilateral regions of interest in the middle cerebellar peduncles (MCP) (3 mm lateral to the fourth ventricle at the mid-fourth ventricle level), hila of the cerebellar nuclei (placed over the center of the nuclei at one image below the level of the MCP voxels), and cerebellar cortex (in the posterolateral aspect of the inferior semilunar lobule on the same image as the cerebellar nuclei voxels, Fig. 1). Regions of interest were confirmed by a single neuroradiologist (AJB). All MRI sequences and head ultrasounds were evaluated for IVH using the grading system of Papile et al. (16), as well as WMI using a scoring system previously shown to be associated with neurodevelopmental outcomes (Mental Development Index of the Bayley Scales of Infant Development II and a verified neuromotor outcome) at $12-18 \mathrm{mo}$ of age (15).

On ultrasound exams, IVH was diagnosed by the presence of hyperechogenic blood layering in the dependent portions of the ventricle or as hyperechogenic clots along the ventricular wall or on the choroid plexuses. On MRI, IVH was diagnosed by the presence of blood layering in the dependent portion of the ventricle or susceptibility artifacts along the ependymal wall within the lateral, third, or fourth ventricle; these were seen as hypointensity in those areas on the T2-weighted spin-echo or echo-planar images. WMI was diagnosed if punctate areas of T1 and mild T2 shortening or more diffuse localized areas of T2 prolongation were identified. A few punctate areas of T1 shortening and marked T2 shortening were noted; these were never seen as isolated findings. They were diagnosed as parenchymal hemorrhage and were considered to be focal WMI. Although T2*-weighted imaging was not acquired due to time considerations, the use of heavily T2-weighted conventional spin echo images (which are much more sensitive to hemorrhage/blood products than the more commonly used fast spin echo sequences) and the analysis of weekly head ultrasound exams should have sensitively detected and grade IVH.

IVH was categorized as mild if there was subependymal hemorrhage or extension into the ventricles without ventricular dilatation, and severe if there was ventricular dilatation or periventricular hemorrhagic infarction. WMI was categorized as mild if there were $\leq 3$ areas of abnormality measuring $<2 \mathrm{~mm}$ and severe if there were $>3$ areas of T1 abnormality or areas $>2 \mathrm{~mm}$ (15).

Linear regression analysis for repeated measures (generalized estimating equation with robust within-subjects correlation estimation) was used to determine the effect of postmenstrual age on cerebellar ADC and FA values, adjusting for the presence of supratentorial IVH and WMI. The effect of the severity of supratentorial brain injury (IVH and WMI) on cerebellar ADC and FA values was explored, correcting for postmenstrual age at the time of the exam. Statistical analysis was performed using Stata 9 (Stata Corporation, College Station, TX).

\section{RESULTS}

Subject cohort. A cohort of 38 preterm neonates was studied with a total of 52 SSFSE-DTI studies. Subject demographics are listed in Table 1. The median gestational age at birth was $27 \mathrm{wk}$ (range, 24-33 wk). The median postmenstrual age at time of imaging was $32 \mathrm{wk}$ for the initial MR scan (range, 27-35 wk) and $36 \mathrm{wk}$ for the second scan (range, 33-40 wk). IVH was identified in 15 scans (four with severe IVH and 11 with mild IVH), whereas WMI was identified in 28 (13 with severe WMI and 15 with mild WMI). No significant difference was found
Table 1. Subject demographics

\begin{tabular}{ll}
\hline & \multicolumn{1}{c}{$\begin{array}{c}\text { Median (range) or } \% \\
\text { incidence }\end{array}$} \\
\hline Gender & 22 males $(58 \%)$ \\
& 16 females $(42 \%)$ \\
Birthweight $(\mathrm{g})$ & $968(590-2260)$ \\
Days intubated $(\mathrm{d})$ & $2(0-100)$ \\
White matter injury & $21 / 38(55 \%)$ \\
Intraventricular hemorrhage & $14 / 38(37 \%)$ \\
Neonatal seizures & $2 / 38(5 \%)$ \\
Patent ductus arteriosus & $10 / 38(26 \%)$ \\
Postnatal infection & $10 / 38(26 \%)$ \\
Ethnicity & 21 White $(53 \%)$ \\
& 9 Hispanic/Latin $(24 \%)$ \\
& 3 Black/African $(8 \%)$ \\
& 3 Asian $(8 \%)$ \\
\hline
\end{tabular}
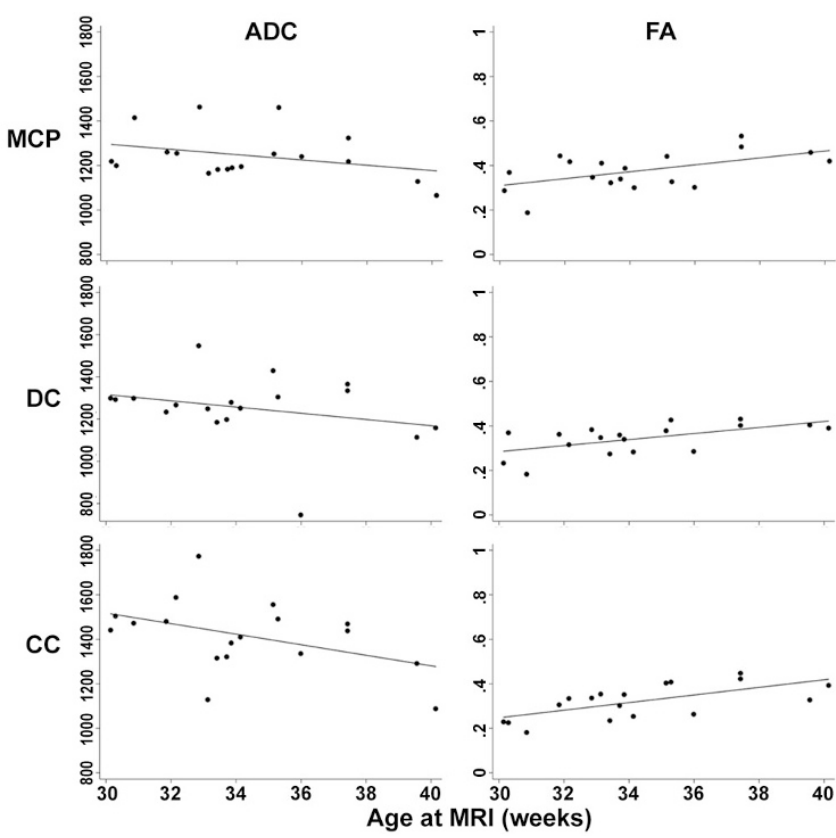

Figure 2. ADC and FA values plotted against postmenstrual age at time of MRI in the 18 scans from newborns without IVH or WMI (linear regression line shown). Units for ADC are $10^{-5} \mathrm{~mm}^{2} / \mathrm{s}$. Regions of interest are labeled as middle cerebellar peduncles (MCP), deep cerebellar nuclei (DC), and cerebellar cortex (CC).

between the ADC or FA values on the left and right in any ROI ( $p>0.05$; Wilcoxon signed-rank test); thus, all further statistical analyses were performed using the average values of both hemispheres. Intrarater reliability was good, with intraclass correlation coefficients of 0.93 for the ADC measurements and 0.92 for the FA measurements. On conventional MRI (including T1- and T2-weighted sequences), the cerebellum seemed normal except for minor punctate cerebellar hemorrhage $(\sim 1 \mathrm{~mm})$ in two patients. Neither patient with minor cerebellar hemorrhage had evidence of supratentorial IVH nor WMI, and the identified areas of hemorrhage were outside of the regions of interest measured.

Effects of gestational age. Linear regression analysis for repeated measures was used to adjust for the presence of supratentorial injury. Adjusting for the presence of supratentorial IVH or WMI, ADC decreased with postmenstrual age at time of imaging in all three regions (all $p<0.01$ ), and FA increased with increasing age in all regions studied (all $p<0.001$ ) (Fig. 2). 
Table 2. Linear regression analysis for ADC and FA in relation to supratentorial IVH and WMI adjusted for gestational age at MRI and postnatal infection

\begin{tabular}{|c|c|c|c|c|c|c|c|c|}
\hline & \multicolumn{2}{|c|}{ Mild IVH } & \multicolumn{2}{|c|}{ Severe IVH } & \multicolumn{2}{|c|}{ Mild WMI } & \multicolumn{2}{|c|}{ Severe WMI } \\
\hline & Coefficient & $p$ & Coefficient & $p$ & Coefficient & $p$ & Coefficient & $p$ \\
\hline \multicolumn{9}{|l|}{ Middle cerebellar peduncles } \\
\hline $\mathrm{ADC}\left(\times 10^{-5} \mathrm{~mm}^{2} / \mathrm{s}\right)$ & 57.8 & 0.3 & 54.3 & 0.01 & -57.2 & 0.03 & -35.6 & 0.5 \\
\hline FA & 0.042 & 0.1 & -0.052 & 0.02 & -0.004 & 0.8 & 0.049 & 0.09 \\
\hline \multicolumn{9}{|l|}{ Deep cerebellar nuclei } \\
\hline $\mathrm{ADC}\left(\times 10^{-5} \mathrm{~mm}^{2} / \mathrm{s}\right)$ & 46.5 & 0.3 & 98.5 & $<0.001$ & -33.9 & 0.3 & -15.9 & 0.8 \\
\hline FA & 0.028 & 0.4 & -0.063 & 0.004 & -0.026 & 0.2 & 0.033 & 0.3 \\
\hline \multicolumn{9}{|l|}{ Cerebellar cortex } \\
\hline $\mathrm{ADC}\left(\times 10^{-5} \mathrm{~mm}^{2} / \mathrm{s}\right)$ & -43.2 & 0.4 & -62.9 & 0.02 & -59.4 & 0.2 & -25.0 & 0.6 \\
\hline FA & 0.044 & 0.2 & -0.051 & 0.007 & -0.016 & 0.5 & 0.039 & 0.1 \\
\hline
\end{tabular}

Coefficients represent the change in measurement per week postmenstrual age seen with supratentorial IVH or WMI.

Effects of concomitant clinical symptoms. Of the documented clinical symptoms, neither the number of days intubated nor the presence of a patent ductus arteriosus correlated with $\mathrm{ADC}$ or FA in any region of interest $(p>0.1)$. Documented postnatal infection was not correlated with ADC values, but was significantly correlated with decreased FA in all regions of interest $(p<0.01)$. Infections documented included sepsis in eight neonates (including one associated with necrotizing enterocolitis), and one neonate each with urinary tract infection and bacterial conjunctivitis. Most cases involved staphylococcal species, in addition to one case each of Citrobacter and Pseudomonas infection. Of the 10 neonates with documented infections, two had both IVH and WMI, four had WMI only, and three had IVH only. All five neonates with IVH had severity categorized as mild. Of the six neonates with WMI, four had severe injury.

Effects of supratentorial injury. Correcting for postmenstrual age and postnatal infection, severe IVH was associated with higher mean diffusivity in the MCP and deep nuclear hila and lower mean diffusivity in the cerebellar cortex (Table 2). Severe IVH was associated with lower FA in all three regions of interest. WMI was not associated with significantly different ADC or FA values, except for the observation that mild WMI was associated with lower mean diffusivity in the MCP.

\section{DISCUSSION}

Using SSFSE-DTI in preterm neonates, maturation of the cerebellum is associated with a decrease in ADC and an increase in FA in the cerebellar cortex, hila of the deep cerebellar nuclei, and white matter of the middle cerebellar peduncle. Severe IVH is associated with disrupted cerebellar microstructure, as reflected in abnormal ADC and FA. Postnatal infection had independent effects on decreasing FA in the cerebellum.

DTI characteristics of preterm neonates without other supratentorial brain injury are reported in this study. Previous studies using DTI have shown that mean diffusivity decreases and FA increases in the supratentorial white matter with increasing age, reflecting maturation of fiber tracts and myelination (12). In contrast, over time, the cerebral cortical gray matter shows increasing mean diffusivity and decreasing FA, reflecting decreasing radial organization (17). The trends found in the cerebellum, in both gray and white matter regions, are similar to those in supratentorial white matter, in keeping with the processes involved in maturation and myelination.

Our analysis of cerebellar diffusivity and anisotropy showed associations with IVH but not as consistently with supratentorial WMI. Independent effects on the cerebellum were also found in relation to postnatal infections. The finding of decreased anisotropy associated with infections suggests relative immaturity, disorganization, or axonal injury in the cerebellum compared with neonates without infections. Previous studies have shown that postnatal sepsis and necrotizing enterocolitis are associated with supratentorial white matter abnormalities (18). Bacterial endotoxins, free radicals, and inflammatory cytokines are some of the proposed causes of this associated brain injury $(19,20)$. This study suggests that injury not only occurs in the supratentorial structures but also in the cerebellum as well.

Early changes in diffusivity and anisotropy in the cerebellum associated with IVH but less clearly in WMI helps to better understand the etiologies of the observed cerebellar volume loss reported in other studies. Multiple volumetric studies of preterm neonates have shown decreased cerebellar volume with supratentorial injury when subjects were imaged from termequivalent age onwards (7-9). Reduced cerebellar volumes in follow-up neuroimaging having been found associated with WMI, IVH with dilatation, and hemorrhagic parenchymal infarction. As well, it has been found that severe unilateral IVH can be associated with contralateral decreased cerebellar volumes in follow-up studies at term-equivalent age $(7,21)$.

Severe supratentorial IVH was found to increase diffusivity and decrease anisotropy in both the MCP and the hila of the deep cerebellar nuclei, while decreasing both diffusivity and anisotropy in the cerebellar cortex. As mean diffusivity decreases and anisotropy increases with increasing age, the high ADC and low FA values associated with IVH suggest delayed maturation or frank injury to the cerebellum and cerebellar peduncles. Because the cerebellar nuclei send their outputs largely through the middle cerebellar peduncle, high ADC values in the MCP and the nuclear hila suggest disrupted development of the normal outgoing connections of the cerebellum associated with severe IVH. This disruption may thus account for the etiology of later cerebellar volume loss.

The diffusivity findings in the cerebellar cortex associated with supratentorial injury varied from that found in the other 
regions of interest. Although we found that cerebellar cortical diffusivity decreased with maturation, similar to the cerebellar nuclear hila and MCP, IVH resulted in differential changes in the cerebellar cortex compared with the other two regions tested. As a result, these findings cannot be explained by delayed maturation. The findings in the cerebellar cortex may reflect the complex nature of this area, including the precursor cells in the external granular layer and the maturing cell layers in the highly folded cerebellar folia. Unfortunately, despite improved cerebellar resolution with DTI-SSFSE, it is still not feasible to perform further tissue-based segmentation of the cerebellar cortex in the largely unmyelinated preterm cerebellum. Further study with animal models may be necessary to elucidate the mechanisms involved.

As WMI is not associated with similar cerebellar diffusion changes, smaller cerebellar volumes may occur more predominantly through later-occurring mechanisms not detected in early DTI. For example, trophic interactions from the damaged supratentorial tissues may result in decreased growth of the contralateral cerebellar hemisphere, as previously suggested in volumetric analyses (7).

The differential effects of IVH and WMI on early cerebellar structural development suggest that hemorrhage may have effects on cerebellar development beyond trophic interactions with supratentorial brain structures. Previous studies have suggested that the surrounding mesenchyme has effects on the development of the cerebellum. Early studies in newborn hamsters showed that destruction of the meningeal cells resulted in disruption of lamination of the cerebellar cortex and decreased cerebellar volumes (22). Additional evidence has emerged in animal models suggesting that cerebellar and cerebral cortical development may be disrupted by mutations of Foxcl, a gene expressed in the meninges but not in the developing brain $(23,24)$, raising the possibility that the malformations caused by Foxcl mutations are related to disturbances in the interactions between the cortex and overlying leptomeninges. Moreover, studies of neurocutaneous melanosis have shown that the presence of abnormal melanincontaining cells in the leptomeninges surrounding the cerebellum results in cerebellar hypoplasia $(25,26)$, supporting the hypothesis of influence of the leptomeninges on cerebellar growth.

Previous retrospective studies on follow-up neuroimaging have found traces of hemosiderin deposition along the external granular layer and periventricular zones in a cohort of children with a history of preterm birth, IVH, and cerebellar volume loss (6). Although only two patients in the present cohort had punctate cerebellar parenchymal hemorrhage detected on conventional MRI, blood in the ventricles and cerebral spinal fluid as a result of IVH, and by extension in the fluid surrounding the cerebellum near the precursor cells of the external granular layer, may have potential negative effects on adjacent cerebellar development. Mechanisms for such effects may either be a direct effect of the presence of hemosiderin or a reflection of disruption of leptomeningeal developmental influence on the underlying cerebellum.

This study uses a novel imaging technique to provide information regarding the effects of gestational age on DTI metrics in the developing cerebellum, as well as the effects of supratentorial injury (in the forms of IVH and WMI), on cerebellar development. Our SSFSE DTI imaging technique overcomes many of the limitations of EPI DTI in assessing the posterior fossa with single channel head coils. As with other neuroimaging studies in neonates, timing of neuroimaging, number of imaging sequences, and choice of patient population are limited by clinical factors including stability of the patient for transport to the MRI facilities. As patients with more severe perinatal insults are more likely to be too clinically unstable for transport, these results in preferential selection of subjects with milder brain injury and systemic illness. Despite these limitations, a number of intriguing correlations were found in this study. Continued neuroimaging of preterm neonates with supratentorial brain injury will help to further characterize these findings.

Early neuroimaging using DTI studies in the posterior fossa can help better understand the mechanisms of cerebellar injury and their relationship to supratentorial injury. This modality may provide an early indicator for cerebellar injury and a predictor for abnormal neurodevelopment in preterm neonates. Ongoing long-term follow-up of these patients will help determine the neurodevelopmental significance of these findings.

\section{REFERENCES}

1. Allin M, Matsumoto H, Santhouse AM, Nosarti C, AlAsady MH, Stewart AL, Rifkin L, Murray RM 2001 Cognitive and motor function and the size of the cerebellum in adolescents born very pre-term. Brain 124:60-66

2. Bodensteiner JB, Johnsen SD 2005 Cerebellar injury in the extremely premature infant: newly recognized but relatively common outcome. J Child Neurol 20:139142

3. Johnsen SD, Bodensteiner JP, Lotze TE 2005 Frequency and nature of cerebellar injury in the extremely premature survivor with cerebral palsy. J Child Neurol 20:60-64

4. Allin MP, Salaria S, Nosaarti C, Wyatt J, Rifkin L, Murray RM 2005 Vermis and lateral lobes of the cerebellum in adolescents born very preterm. Neuroreport $16: 1821-1824$

5. Mercuri E, He J, Curati WL, Dubowitz LM, Cowan FM, Bydder GM 1997 Cerebellar infarction and atrophy in infants and children with a history of premature birth. Pediatr Radiol 27:139-143

6. Messerschmidt A, Brugger PC, Boltshauser E, Zoder G, Sterniste W, Birnbacher R, Prayer D 2005 Disruption of cerebellar development: potential complication of extreme prematurity. AJNR Am J Neuroradiol 26:1659-1667

7. Limperopoulos C, Soul JS, Haider H, Huppi PS, Bassaran H, Warfield SK, Robertson RL, Moore M, Akins P, Volpe JJ, du Plessis AJ 2005 Impaired trophic interactions between the cerebellum and the cerebrum among preterm infants. Pediatrics 116:844-850

8. Shah DK, Anderson PJ, Carlin JP, Pavlovic M, Howard K, Thompson DK 2006 Reduction in cerebellar volumes in preterm infants: relationship to white matter injury and neurodevelopment at two years of age. Pediatr Res 60:97-102

9. Srinivasan L, Allsop J, Counsell SJ, Boardman JP, Edwards AD, Rutherford M 2006 Smaller cerebellar volumes in very preterm infants at term-equivalent age are associated with the presence of supratentorial lesions. AJNR Am J Neuroradiol 27:573-579

10. Huppi PS, Maier SE, Peled S, Zientara GP, Barnes PD, Jolesz FA, Volpe JJ 1998 Microstructural development of human newborn cerebral white matter assessed in vivo by diffusion tensor magnetic resonance imaging. Pediatr Res 44:584-590

11. Neil JJ, Shiran SI, McKinsry RC, Schefft GL, Snyder AZ, Almli CR, Akbudak E, Aronovitz JA, Miller JP, Lee BC, Conturo TE 1998 Normal brain in human newborns: apparent diffusion coefficient and diffusion anisotropy measured by using diffusion tensor MR imaging. Radiology 209:57-66

12. Miller SP, Vigneron DB, Henry RG, Bohland MA, Ceppi-Cozzio C, Hoffman C, Newton N, Partridge JC, Ferriero DM, Barkovich AJ 2002 Serial quantitative diffusion tensor MRI of the premature brain: development in newborns with and without injury. J Magn Reson Imaging 16:621-632

13. Xu D, Henry RG, Mukherjee P, Carvajal L, Miller SP, Barkovich AJ, Vigneron DB 2004 Single-shot fast spin-echo diffusion tensor imaging of the brain and spine with head and phased array coils at 1.5T and 3.0T. Magn Reson Imaging 22:751-775

14. Partridge SC, Mukherjee P, Berman JI, Henry RG, Miller SP, Lu U, Glenn OA, Ferriero DM, Barkovich AJ, Vigneron DB 2005 Tractography-based quantitation of diffusion tensor imaging parameters in white matter tracts of preterm newborns. J Magn Reson Imaging 22:467-474 
15. Miller SP, Ferriero DM, Leonard C, Piecuch R, Glidden DV, Partridge JC, Perez M, Mukherjee P, Vigneron DB, Barkovich AJ 2005 Early brain injury in premature newborns detected with magnetic resonance imaging is associated with adverse early neurodevelopmental outcome. J Pediatr 147:609-616

16. Papile LA, Burstein J, Burstein R, Koffler H 1978 Incidence and evolution of subependymal and intraventricular hemorrhage: a study of infants with birth weights less than 1,500 gm. J Pediatr 92:529-534

17. McKinstry RC, Mathur A, Miller JH, Ozcan A, Snyder AZ, Schefft GL, Almli CR, Shiran SI, Conturo TE, Neil JJ 2002 Radial organization of developing preterm human cerebral cortex revealed by non-invasive water diffusion anisotropy MRI. Cereb Cortex 12:1237-1243

18. Shah DK, Doyle LW, Anderson PJ, Bear M, Daley AJ, Hunt RW, Inder TE 2008 Adverse neurodevelopment in preterm infants with postnatal sepsis or necrotizing enterocolitis is mediated by white matter abnormalities on magnetic resonance imaging at term. J Pediatr 153:170-175, 175.e1

19. Volpe JJ 2001 Neurobiology of periventricular leukomalacia in the premature infant. Pediatr Res 50:553-562

20. Duncan JR, Cock ML, Scheerlinck JP, Westcott KT, McLean C, Harding R, Rees SM 2002 White matter injury after repeated endotoxin exposure in the preterm ovine fetus. Pediatr Res 52:941-949
21. Rollins NK, Wen TS, Dominquez R 1995 Crossed cerebellar atrophy in children: a neurologic sequela of extreme prematurity. Pediatr Radiol 25:S20-S25

22. Von Knebel Doeberitz C, Sievers J, Sadler M, Pehlemann FW, Berry M, Halliwell P 1986 Destruction of meningeal cells over the newborn hamster cerebellum with 6-hydroxydopamine prevents foliation and lamination in the rostral cerebellum. Neuroscience 17:409-426

23. Maclean K, Smith J, St Heaps L, Chia N, Williams R, Peters GB, Onikul E, McCrossin T, Lehmann OJ, Ades LC 2005 Axenfeld-Rieger malformation and distinctive facial features: clues to a recognizable $6 \mathrm{p} 25$ microdeletion syndrome. Am J Med Genet A 132:381-385

24. Zarbalis K, Siegenthaler JA, Choe Y, May SR, Peterson AS, Pleasure SJ 2007 Cortical dysplasia and skull defects in mice with a Foxc1 allele reveal the role of meningeal differentiation in regulating cortical development. Proc Natl Acad Sci USA 104:14002-14007

25. Barkovich AJ, Frieden IJ, Williams ML 1994 MR of neurocutaneous melanosis. AJNR Am J Neuroradiol 15:859-867

26. Kadonaga JN, Barkovich AJ, Edwards MS, Frieden IJ 1992 Neurocutaneous melanosis in association with the Dandy-Walker complex. Pediatr Dermatol 9:37-43 